and our data in this regard will be helpful to readers for further understanding the pathology of MCCs.

\section{Acknowledgments}

This study was supported, in part, by a grant from the Japan Society for the Promotion of Science (No. 26460433).

\section{Disclosure/conflict of interest}

The authors declare no conflict of interest.

Takeshi Iwasaki ${ }^{1,4}$, Michiko Matsushita1,2,4, Daisuke Nonaka ${ }^{3}$, Ichiro Murakami ${ }^{1}$ and Kazuhiko Hayashi ${ }^{1}$

${ }^{1}$ Division of Molecular Pathology, Department of Pathology, Tottori University Faculty of Medicine, Yonago, Japan; ${ }^{2}$ Department of Pathobiological Science and Technology, School of Health Science,

Tottori University Faculty of Medicine, Yonago, Japan; ${ }^{3}$ Department of Histopathology, The Christie NHS Foundation Trust, Manchester, UK E-mail: iwasakit-path@umin.ac.jp or hayashik@med.tottori-u.ac.jp ${ }^{4}$ These authors contributed equally to this work.

\section{References}

1 Miner AG, Patel RM, Wilson DA et al. Cytokeratin 20-negative Merkel cell carcinoma is infrequently associated with the Merkel cell polyomavirus. Mod Pathol 2014;28:498-504.

2 Scott MP, Helm KF. Cytokeratin 20: a marker for diagnosing Merkel cell carcinoma. Am J Dermatopathol 1999;21:16-20.

3 Matsushita M, Iwasaki T, Kuwamoto S et al. Merkel cell polyomavirus (MCPyV) strains in Japanese merkel cell carcinomas (MCC) are distinct from Caucasian type MCPyVs: genetic variability and phylogeny of MCPyV genomes obtained from Japanese MCPyVinfected MCCs. Virus Genes 2014;48:233-242.

4 Laude HC, Jonchere B, Maubec E et al. Distinct merkel cell polyomavirus molecular features in tumour and non tumour specimens from patients with merkel cell carcinoma. PLoS Pathog 2010;6:e1001076.

$5 \mathrm{Kim} \mathrm{JH}$, Rhee YY, Bae JM et al. Loss of CDX2/CK20 expression is associated with poorly differentiated carcinoma, the CpG island methylator phenotype, and adverse prognosis in microsatellite-unstable colorectal cancer. Am J Surg Pathol 2013;37:1532-1541.

6 Iwasaki T, Matsushita M, Kuwamoto S et al. Usefulness of significant morphologic characteristics in distinguishing between Merkel cell polyomavirus-positive and Merkel cell polyomavirus-negative Merkel cell carcinomas. Hum Pathol 2013;44:1912-1917.

\title{
Reply to Commentary on "Cytokeratin 20-negative Merkel cell carcinoma is infrequently associated with the Merkel cell polyomavirus"
}

Modern Pathology (2016) 29, 90-91; doi:10.1038/modpathol.2015.125

To the editor: We appreciate the comments by Iwasaki et al regarding our recent article, 'Cytokeratin 20-negative Merkel cell carcinoma is infrequently associated with the Merkel cell polyomavirus.' They report findings in an independent cohort of cytokeratin 20-negative Merkel cell carcinomas (MCCs), the majority of which are negative for Merkel cell polyomavirus (MCPyV), in agreement with our observations.

Among MCCs classified as MCPyV positive in our study, one case (\#13) had relatively low MCPyV by quantitative PCR (qPCR). Although qPCR is accepted as the gold standard for MCPyV detection in MCC, there is debate about whether low levels of MCPyV represent tumorigenic virus or contamination by background wild-type virus. ${ }^{1-3}$ In addition, the sensitivity of any given primer pair may vary dramatically from case to case. ${ }^{1}$ Hence, currently there is no universally accepted threshold for considering a tumor MCPyV positive by qPCR. We agree with Iwasaki et al that immunohistochemistry for MCPyV large $\mathrm{T}$ antigen (LTAg), while less sensitive than qPCR, may be informative in some cases that are borderline by qPCR. We performed immunohistochemistry for LTAg expression in case \#13 using CM2B4 antibody as previously described. ${ }^{4}$ This demonstrated moderate to strong nuclear staining for LTAg in $>80 \%$ of tumor cells, validating our classification of this tumor as MCPyV positive.

We agree that loss of cytokeratin-20 expression may be associated with loss of differentiation in MCC. However, further study is needed to determine the molecular similarity of these tumors to conventional (cytokeratin 20-positive) MCC.

Andrew G Miner ${ }^{1,2}$, Rajiv M Patel ${ }^{3,4}$, Deborah Wilson ${ }^{2}$, Gary W Procop ${ }^{2}$, Eugen Minca ${ }^{2}$, Douglas R Fullen ${ }^{3,4}$, Paul W Harms ${ }^{3,4}$ and Steven D Billings ${ }^{2}$

${ }^{1}$ Cleveland Clinic Department of Dermatology, Cleveland, OH, USA; ${ }^{2}$ Cleveland Clinic Department of Pathology, Cleveland, OH, USA; ${ }^{3}$ Department of Pathology, University of Michigan, Ann Arbor, MI, 
USA; ${ }^{4}$ Department of Dermatology, University of Michigan, Ann Arbor, MI, USA E-mail: billins@ccf.org

\section{References}

1 Rodig SJ, Cheng J, Wardzala J et al. Improved detection suggests all Merkel cell carcinomas harbor Merkel polyomavirus. J Clin Invest 2012;122:4645-4653.

2 Matsushita M, Iwasaki T, Kuwamoto S et al. Merkel cell polyomavirus (MCPyV) strains in Japanese merkel cell carcinomas (MCC) are distinct from Caucasian type MCPyVs: genetic variability and phylogeny of $\mathrm{MCPyV}$ genomes obtained from Japanese MCPyVinfected MCCs. Virus Genes 2014;48:233-242.

3 Bhatia K, Goedert JJ, Modali R et al. Merkel cell carcinoma subgroups by Merkel cell polyomavirus DNA relative abundance and oncogene expression. Int J Cancer 2010;126:2240-2246.

4 Fisher CA, Harms PW, McHugh JB et al. Small cell carcinoma in the parotid harboring Merkel cell polyomavirus. Oral Surg Oral Med Oral Pathol Oral Radiol 2014;118:703-712.

\section{NRASQ61R immunohistochemistry: a new tool for mutational status screening in challenging melanoma samples}

Modern Pathology (2016) 29, 91-92; doi:10.1038/modpathol.2015.78

To the editor: We read with interest the recent study by Massi et $a l^{1}$ concerning the evaluation of the new anti-human N-Ras (Q61R) monoclonal antibody (clone SP174) in the mutational screening of melanoma samples. As the recently described BRAFV600E mutation-specific immunohistochemistry, an antiNRASQ61R mutation-specific antibody may consist of a cost-effective and faster ancillary tool in the mutational screening process that has now become a major requirement for the management of patients with metastatic melanoma. The sensitivity and specificity of $100 \%$ reported in this study are very encouraging although they need to be confirmed by additional studies as mentioned by the authors, especially in metastatic samples. In our opinion, some other technical points are worth to be notified.

First, we want to point out the importance of the characteristics of melanoma samples. The authors have only selected primary melanomas with Breslow thickness over $4 \mathrm{~mm}$, although many melanomas have a far thinner Breslow thickness. No data is provided concerning the percentage of tumoral cells in the samples or the modalities of DNA extraction from formalin-fixed and paraffin-embedded tissues used for molecular genetic analysis. Notably, the use of macro- or micro-dissection based on histopathological examination of hematoxylin and eosin-stained sections is not mentioned. The percentage of tumoral cells in the samples and the mutated allele proportion in the extracted DNA can highly influence the results of molecular analysis and the ability of the genotyping method to detect a mutation. In our opinion, these parameters have to be taken into account when interpreting a molecular mutational status result. ${ }^{2}$ If we keep in mind the thicknesses over $4 \mathrm{~mm}$ of the melanomas analyzed in this study, we can postulate that all samples contained a great proportion of tumoral cells. Nevertheless, we cannot rule out the hypothesis that a high amount of inflammatory reactive cells in some samples can decrease the relative amount of tumor in the extracted DNA. Another major interest of mutation-specific immunohistochemistry is the ability to stain a minority of tumoral cells within a sample containing a majority of nontumoral cells (ie, thin primary melanoma or lymph node micrometastasis). ${ }^{3}$ These data are relevant for diagnosis and could explain that one sample in the study by Massi et al that was initially considered as NRAS wild type by molecular analysis was indeed positive for anti-NRASQ61R immunolabeling and finally found to be $N R A S^{Q 61 R}$ mutated after molecular reanalysis.

Second, the authors analyzed 97 samples as the last sample of their series was excluded because of amplification failure preventing molecular analysis. In our experience, another advantage of mutationspecific antibodies is the opportunity of bringing out a mutated protein in samples that are not conclusive using genotyping methods (about $2-3 \%$ of samples in our experience, unpublished data). The immunohistochemical analysis of this remaining sample could have been of interest, despite the fact that, in case of negativity, an NRAS mutation could not have been definitively ruled out.

Third, the authors defined the cutoff for positivity at $60 \%$ or more of viable tumoral cells with moderate to strong immunelabeling intensity. Weaker labeling and/or single interspersed immunostained cells were considered as negative and nonspecific. This fact points out the real difficulty in identifying melanoma cells versus histiocytic/macrophagic cells. We agree with Massi et al that the interpretation of isolated NRASQ61R- or BRAFV600E-immunolabeled cells can be difficult, and sometimes not conclusive especially in case of florid reactive inflammatory infiltrate. Nevertheless, it is still not perfectly clear whether 